\title{
GERONTOTECNOLOGIA NA PROMOÇÃO DO CUIDADO PALIATIVO: UMA REVISÃO INTEGRATIVA ${ }^{1}$
}

\author{
GERONTOTECHNOLOGY IN THE PROMOTION OF PALLIATIVE CARE: \\ AN INTEGRATIONAL REVIEW
}

\author{
Mariele Gomes de Oliveira² e Maria Helena Gehlen ${ }^{3}$
}

\section{RESUMO}

Objetiva-se descrever como se desenvolve a produção de cuidados paliativos da pessoa idosa ao aliar a gerontotecnologia ao atendimento multiprofissional. Realizou-se uma revisão integrativa de literatura e, a partir da síntese do conteúdo dos artigos, foi possível analisar as produções e construir dois eixos temáticos como guia de discussão denominados: A pessoa idosa e a promoção do cuidado paliativo; e (Bio)gerontotecnologia: uma necessidade à promoção do cuidado paliativo. Nesse sentido, é preciso criar uma cultura de cuidados paliativos para melhorar a qualidade de vida, promovendo o suporte clínico, humano e emocional. Além disso, o grande potencial para inovação da gerontotecnologia e a crescente difusão dos cuidados paliativos leva a crer que a (bio)gerontotecnologia é uma ferramenta importante para o cuidado paliativo, sendo possível potencializar a produção ou a aquisição de dispositivos, produtos e instrumentos de cuidado.

Palavras-chave: Idoso, Cuidado paliativo, Gerontotecnologia.

\begin{abstract}
It is aimed at describing the way the palliative care production in the elderly develops when combining gerontotechnology to the multiprofessional care. An integrative literature review was performed and from the synthesis of the content of the articles, it was possible to perform the analysis of the productions and build two thematic axes as a discussion guide entitled as: The elderly and the promotion of palliative care and; (Bio) gerontotechnology: a necessity for the promotion of palliative care. In this sense, it is necessary to create a culture of palliation to improve the quality of life, promoting clinical, human and emotional support. In addition, the great potential for innovation in gerontotechnology and the growing diffusion of palliative care leads us to believe that (bio) gerontotechnology is an important tool for palliative care and it is possible to enhance the production or acquisition of care devices, products and instruments.
\end{abstract}

Keywords: Elderly, Palliative care, Gerontotechnology.

\footnotetext{
${ }^{1}$ Estudo oriundo da especialização em Terapia Intensiva: Ênfase em Oncologia e Controle de Infecção Hospitalar.

${ }^{2}$ Acadêmica do Curso de Pós-Graduação em Terapia Intensiva da Universidade Franciscana. E-mail: marieleg.deoliveira@ gmail.com.

${ }^{3}$ Orientadora. Docente da Universidade Franciscana. Enfermeira. Especialista em Enfermagem na Saúde do Idoso. Especialista em Educação Inclusiva. Mestre em Educação. Doutora em Gerontologia Biomédica. E-mail: gehlenmmh@gnail.com.
} 


\section{INTRODUÇÃO}

O Instituto Brasileiro de Geografia e Estatística (2013) estima que o número de pessoas com mais de 65 anos é de, aproximadamente, 1 a cada 10 brasileiros. Em 2060, 1 a cada 4 brasileiros vai estar nessa faixa etária, o que demonstra que a demografia brasileira tem sofrido mudanças nas últimas décadas, principalmente quanto à inversão da pirâmide etária, colocando os idosos em maior número. Esse aumento da população idosa é devido à redução da natalidade, fazendo com que o nível de idosos cresça proporcionalmente.

No processo de envelhecimento, a pessoa idosa poderá estar sujeita a condições clínicas críticas que requerem atendimento multiprofissional e são oriundas de patologias, tais como câncer, doenças osteomusculares e neurológicas crônicas, as quais acarretam dependência funcional para a realização de atividades básicas, que, junto ao declínio da condição de saúde, passam a necessitar de cuidados paliativos (SILVEIRA; CIAMPONE; GUTIERREZ, 2014).

O envelhecimento humano com suas manifestações, características da senescência, poderá se relacionar com a senilidade ocasionando à pessoa idosa dependência para a realização de suas atividades, principalmente, quando necessita dos cuidados paliativos. Desse modo, as descobertas na Ciência buscam a elaboração de novas tecnologias, substâncias farmacológicas e não farmacológicas que possibilitem a promoção de cuidado paliativo, sem sofrimento, confrontando as patologias (CIOSAK et al., 2011; FLORES; BENVEGNÚ, 2008).

A Organização Mundial de Saúde (OMS) descreve os cuidados paliativos como os serviços destinados a prevenir e aliviar o sofrimento de pacientes e famílias que enfrentam doenças que ameaçam a vida, por meio do gerenciamento precoce da dor e de outros problemas físicos, psicossociais e espirituais. Estudiosos sobre o tema enfatizam a importância da prática de tais cuidados por meio de equipe multiprofissional e defendem que os cuidados paliativos visam aprimorar a qualidade de vida de pacientes e de seus familiares (SILVEIRA; CIAMPONE; GUTIERREZ, 2014).

Tendo em vista que os cuidados paliativos visam primordialmente à promoção da qualidade de vida, torna-se imprescindível que a equipe multiprofissional busque aprimorar seus conhecimentos na área de domínio do conhecimento em gerontologia e suas tecnologias. A gerontotecnologia por ser um campo de estudo interdisciplinar e reunir esses pilares possibilita a promoção de cuidado paliativo da pessoa idosa em condições clínicas críticas, alocando a tecnologia e a gerontologia na humanização da assistência prestada (BOUMA et al., 2007).

Nesse sentido, apresenta-se a questão de pesquisa: quais as evidências científicas na literatura nacional e internacional efetivam a gerontotecnologia na promoção dos cuidados paliativos da pessoa idosa? Ainda, tem-se como objetivo descrever como se desenvolve a promoção de cuidados paliativos da pessoa idosa ao aliar a gerontotecnologia ao atendimento multiprofissional. 


\section{MATERIAIS E MÉTODOS}

Por meio das etapas proposta por Rother (2007), realizou-se uma revisão integrativa de literatura, constituída pelas seguintes etapas: identificação da questão de pesquisa, estabelecimento dos descritores, recorte temporal, busca da amostragem na literatura, coleta de dados, análise dos estudos incluídos e, por fim, resultados e discussão da síntese do conteúdo.

Para tanto, foram considerados os artigos publicados e indexados na biblioteca virtual em saúde (BVS), em bancos de dados eletrônicos da Literatura Latino-Americana e do Caribe em Ciências da Saúde (LILACS) e do Medical Literature Analysis and Retrievel System Online (MEDLINE). Os descritores foram estabelecidos e classificados em Ciências da Saúde (DeCS) e refinados utilizando-se o operador booleano "and".

No que se refere à avaliação do nível de evidência, os estudos foram classificados de acordo com a definição dos autores Melnyk e Fineout-Overholt (2005) como nível V, não havendo um recorte temporal estabelecido. Foram considerados artigos em português, espanhol, inglês, bem como os de livre acesso e, por outro lado, foram excluídos aqueles que não versaram sobre o tema e também aqueles que se repetiram nos bancos de dados. A estratégia utilizada para a identificação dos artigos pode ser vista na Figura 1, representada pelo fluxograma da seleção da amostra.

Figura 1 - Fluxograma da seleção da amostra

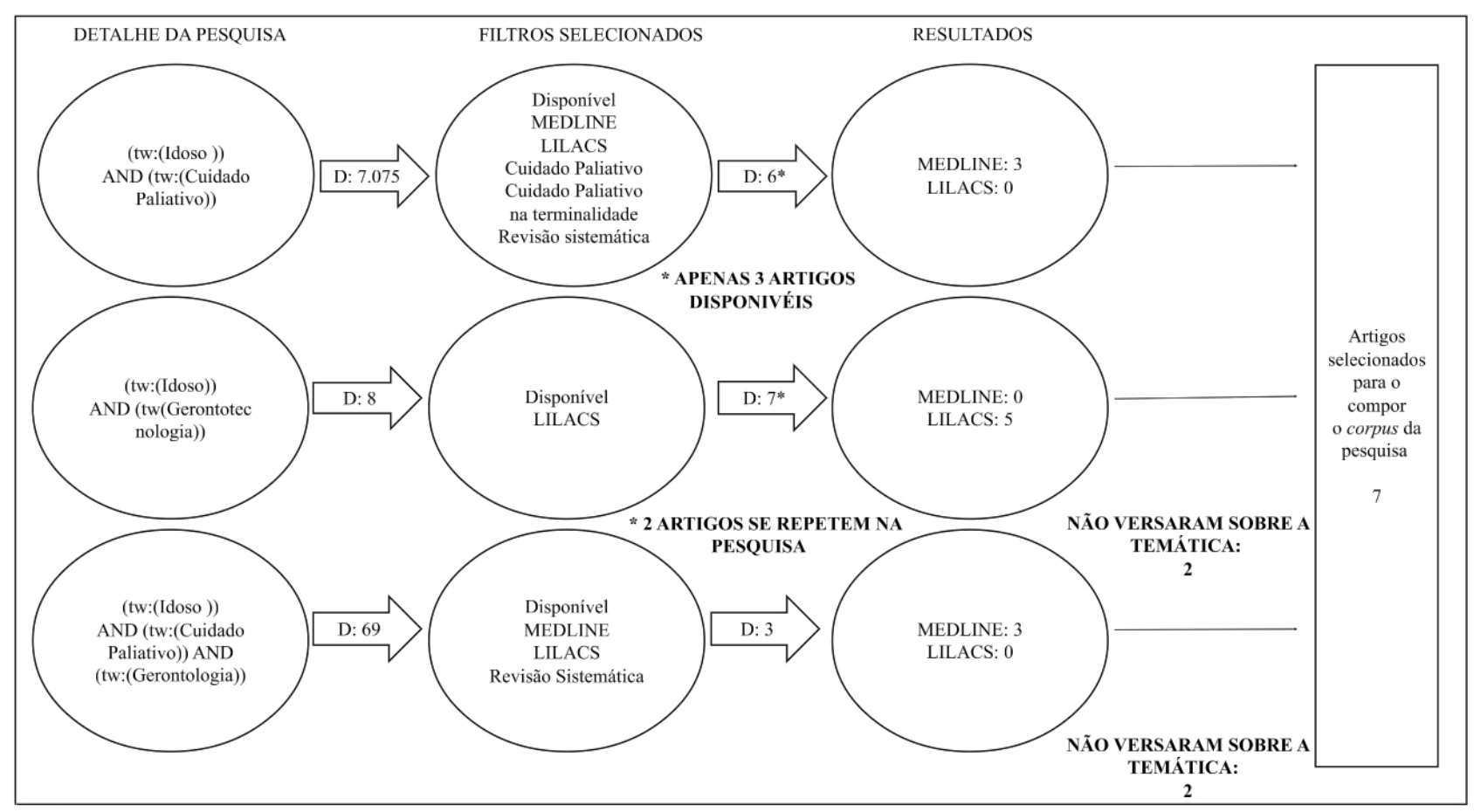

Fonte: Elaborada pela Autora (2019). 
Dessa forma, obteve-se o montante de 7 (sete) achados para compor o corpus de análise do estudo.

No intuito de facilitar a compreensão dos achados metodológicos, ou seja, da forma como foram conduzidos os estudos frente à literatura científica, e com vistas a discuti-los, foi elaborada uma tabela denominada "referências da integrativa dos artigos", conforme demonstrado na Tabela 1.

Tabela 1 - Referências da integrativa dos artigos

\begin{tabular}{|c|c|c|c|c|}
\hline $\begin{array}{c}\text { Código } \\
\text { do estudo }\end{array}$ & Título & Periódico/ano & $\begin{array}{c}\text { Delineamento da } \\
\text { pesquisa/ nível de } \\
\text { evidência }\end{array}$ & Principais resultados \\
\hline A1 & $\begin{array}{l}\text { Realizing a palliative } \\
\text { approach in dementia } \\
\text { care: strategies to faci- } \\
\text { litate aged care staff } \\
\text { engagement in evidence- } \\
\text {-based practice }\end{array}$ & $\begin{array}{l}\text { Int Psychoge- } \\
\text { riatrics. } 2009\end{array}$ & $\begin{array}{l}\text { Pesquisa Qualitativa. } \\
\text { Nível V }\end{array}$ & $\begin{array}{l}\text { Por meio de seu engajamento em ciclos } \\
\text { sucessivos de pesquisa de ação, essa } \\
\text { equipe acessou recursos baseados em } \\
\text { evidências e desenvolveu estratégias para } \\
\text { atender às necessidades de informação } \\
\text { dos membros da família. }\end{array}$ \\
\hline A2 & $\begin{array}{l}\text { End of life care for } \\
\text { community dwell- } \\
\text { ing older people with } \\
\text { dementia: an integrated } \\
\text { review }\end{array}$ & $\begin{array}{l}\text { Int J Geriatr } \\
\text { Psychiatry. } \\
2010\end{array}$ & $\begin{array}{l}\text { Pesquisa Qualitativa. } \\
\text { Nível V }\end{array}$ & $\begin{array}{l}\text { Os poucos estudos que desenvolveram } \\
\text { ferramentas específicas para demência } \\
\text { nos cuidados de final de vida e medidas } \\
\text { de resultados específicos para melhorar o } \\
\text { conforto e a comunicação demonstraram } \\
\text { o que poderia ser alcançado e quanto mais } \\
\text { precisa ser feito. }\end{array}$ \\
\hline A3 & $\begin{array}{l}\text { Informed palliative } \\
\text { care in nursing homes } \\
\text { through the inter- } \\
\text { RAI Palliative Care } \\
\text { instrument:a study } \\
\text { protocol based on the } \\
\text { Medical Research } \\
\text { Council framework }\end{array}$ & $\begin{array}{l}\text { BMC Geriatrics. } \\
2014\end{array}$ & $\begin{array}{l}\text { Pesquisa Qualitativa. } \\
\text { Nível V }\end{array}$ & $\begin{array}{l}\text { Este é o primeiro estudo a avaliar a } \\
\text { validade e o efeito do PC interRAI em } \\
\text { casas de repouso, seguindo uma metodo- } \\
\text { logia baseada na estrutura do MRC. Essa } \\
\text { abordagem melhora o desenho do estudo } \\
\text { e implementação e contribui para maior } \\
\text { generalização dos resultados. }\end{array}$ \\
\hline A4 & $\begin{array}{l}\text { (Geronto)Tecnologia } \\
\text { cuidativo-educacional } \\
\text { na doença de Alzheimer } \\
\text { e no apoio ao idoso/ } \\
\text { família: perspectiva dos } \\
\text { docentes e discentes }\end{array}$ & $\begin{array}{l}\text { Escola Anna } \\
\text { Nery. } 2017\end{array}$ & $\begin{array}{l}\text { Pesquisa exploratória, } \\
\text { descritiva, qualitativa. } \\
\text { Nível IV }\end{array}$ & $\begin{array}{l}\text { Docentes e discentes compreendem o grupo } \\
\text { como uma (geronto)tecnologia, pelas ações } \\
\text { de educação e cuidado desenvolvidas. } \\
\text { Referem possuir a capacidade de produzir } \\
\text { novas gerontotecnologias, o que demonstra } \\
\text { a sua capacidade como (geronto)tecnologia } \\
\text { cuidativo-educacional complexa. }\end{array}$ \\
\hline A5 & $\begin{array}{l}\text { (Geronto)tecnologia } \\
\text { cuidativo-educacional } \\
\text { complexa para pessoas } \\
\text { idosas/famílias com a } \\
\text { doença de Alzheimer }\end{array}$ & $\begin{array}{l}\text { Rev Bras } \\
\text { Enferm. } 2017\end{array}$ & $\begin{array}{l}\text { Pesquisa exploratória, } \\
\text { descritiva, qualitativa. } \\
\text { Nível IV }\end{array}$ & $\begin{array}{l}\text { Grupo contribui como (geronto)tecnologia } \\
\text { de cuidado e educação para cuidar. Nele } \\
\text { são construídos conhecimentos que, apli- } \\
\text { cados na prática, auxiliam nas desordens } \\
\text { vivenciadas, melhorando o cuidado da } \\
\text { pessoa idosa com doença de Alzheimer. }\end{array}$ \\
\hline
\end{tabular}




\begin{tabular}{|c|c|c|c|c|}
\hline A6 & $\begin{array}{l}\text { Jogo de tabuleiro: uma } \\
\text { gerontotecnologia na } \\
\text { clínica do cuidado de } \\
\text { enfermagem }\end{array}$ & $\begin{array}{l}\text { Rev Bras } \\
\text { Enferm. } 2018\end{array}$ & $\begin{array}{l}\text { Pesquisa qualitativa. } \\
\text { Nível IV }\end{array}$ & $\begin{array}{l}\text { A gerontotecnologia implementada na clí- } \\
\text { nica do cuidado de enfermagem agiu como } \\
\text { elemento lúdico no exercício da autodeter- } \\
\text { minação e independência do idoso, como } \\
\text { potencializador de memória, autoestima, } \\
\text { processos de socialização, trocas de expe- } \\
\text { riências e aprendizagem compartilhada. }\end{array}$ \\
\hline A7 & $\begin{array}{l}\text { Geriatric Emergency } \\
\text { Department Guidelines }\end{array}$ & $\begin{array}{l}\text { Annals of } \\
\text { Emergency } \\
\text { Medicine. } 2014\end{array}$ & $\begin{array}{l}\text { Pesquisa Qualitativa, } \\
\text { Nível V }\end{array}$ & $\begin{array}{l}\text { Essas diretrizes criam um modelo para o } \\
\text { pessoal, equipamentos, educação, políticas } \\
\text { de procedimentos, cuidados ao acom- } \\
\text { panhamento e medidas de melhoria de } \\
\text { desempenho. }\end{array}$ \\
\hline
\end{tabular}

Fonte: Oliveira (2019).

De posse dos artigos selecionados, procedeu-se à leitura criteriosa deles, organizando-os pela inicial A, acompanhada de numeração. Então, com a base de dados constituída, realizou-se a síntese do conteúdo das produções e, sequencialmente, procedeu-se à análise e à discussão da temática, de acordo com Minayo (2012).

A partir da síntese do conteúdo dos artigos foi possível realizar a análise das produções e construir dois eixos temáticos como guia de discussão denominados:

- A pessoa idosa e a promoção do cuidado paliativo;

- (Bio)gerontotecnologia: uma necessidade à promoção do cuidado paliativo.

\section{RESULTADOS E DISCUSSÃO}

A pessoa idosa e a promoção do cuidado paliativo

A1 evidenciou que a equipe de saúde e os familiares que promoviam o cuidado da pessoa idosa apresentavam pouca compreensão das evidências crescentes dos benefícios de uma abordagem paliativa. Além disso, perceberam que quando se tem a oportunidade de refletir e criticar o desenvolvimento do cuidado paliativo, por meio das evidências, é possível à equipe de atenção à idade estar melhor posicionada para adotar uma abordagem paliativa e, então, executar mudanças em sua prática para melhorar a provisão de cuidados.

Segundo Gutierrez e De Barros (2012), Cuidados Paliativos são aqueles prestados aos pacientes considerados fora de possibilidade de cura, cuja doença tem poucas chances de resposta positiva à terapêutica curativa, sendo imprescindível, nessa fase, o controle da dor e o alívio do sofrimento em todas as dimensões do ser humano, sejam elas físicas, psíquicas, sociais e espirituais. Desse modo, o cuidado deve ser diferenciado, individualizado, levando-se em consideração a singularidade e a necessidade de cada pessoa que se encontra em condição de dependência.

Portanto, acredita-se que a equipe multiprofissional deve apropriar-se das evidências científicas, bioéticas, humanas e tecnológicas na promoção do cuidado, envolvendo a família de maneira a 
construir a autonomia na tomada de decisão e na efetividade da paliação, de acordo com as necessidades educativas, espirituais, clínicas e críticas da pessoa idosa.

Nesse contexto, A2 demonstra que os cuidados da pessoa idosa, com ou sem agravos associados ao seu processo de envelhecimento, necessitam de evidências que apoiam o uso de ferramentas tecnológicas em casas/lares com acesso intermitente do suporte de cuidados paliativos especializados. Ainda, é reforçada a importância da equipe multiprofissional no planejamento e no atendimento das necessidades da pessoa idosa e de sua família.

Salienta-se que nesse estudo houve limitações quanto aos resultados esperados, devido à ausência de compreensão compartilhada de como o fim da vida é reconhecido, ou em que ponto o fim da vida é definido para pessoas idosas.

Sob esse viés, Forbes, Bern-Klug e Gessert (2000) observaram que é fundamental uma compreensão também do cenário familiar, pois a família necessita de assistência da equipe multiprofissional para processar emoções difíceis e dolorosas, frente à trajetória da doença e à avaliação das decisões que facilitam ou dificultam uma morte natural, compreendida durante o desenvolvimento do cuidado paliativo.

A3 corrobora os demais estudos já descritos ao apresentar uma revisão sistemática para desenvolver um protocolo que permite a avaliação da funcionalidade em cuidado paliativo, ressaltando que o atendimento geriátrico-gerontológico atende às necessidades dos idosos. Vale ressaltar que se trata de um estudo longitudinal, ainda em andamento. De tal modo, os dados parciais da pesquisa viabilizam a avaliação funcional em cuidados paliativos, sendo esta de suma importância para a equipe traçar um plano de atendimento humanizado e integral, adequado para a população idosa.

Acredita-se na necessidade da real efetividade da promoção de cuidados paliativos no que se referem Sanvezzo, Montandon e Esteves (2018) ao mencionarem que os instrumentos de avaliação de funcionalidade de idosos em cuidados paliativos são fundamentais para a vigilância e evolução da doença, pois tais instrumentos constituem elemento valioso na tomada de decisões, no prognóstico, no diagnóstico e na prevenção de efeitos adversos associados ao declínio funcional. No entanto, apesar da importância do status funcional para os desfechos de saúde, os dados sobre funcionalidade raramente são coletados durante a assistência de rotina.

Ao integrar os estudos que dizem respeito à promoção do cuidado paliativo da pessoa idosa, emergiram as evidências científicas para a construção da segunda categoria intitulada: (bio)gerontotecnologia: uma necessidade à promoção do cuidado paliativo.

(Bio)gerontotecnologia: uma necessidade à promoção do cuidado paliativo.

Segundo A4, o termo "(geronto)tecnologia cuidativo-educacional complexa" derivou-se da união dos conceitos de tecnologia, gerontologia e complexidade. Trata-se de produto, processo, estratégias, serviço e/ou conhecimento, com a finalidade cuidativo-educacional da pessoa idosa e de seus familiares/cuidadores, fruto de uma construção/vivência coletiva complexa, que 
valoriza as relações, interações e retroações dos envolvidos, por meio do conhecimento inter-multi-trans-meta-disciplinar.

Barros et al. (2012) refere-se à gerontotecnologia como derivada dos conceitos de gerontologia e tecnologia, entendidas como tecnologias contributivas para o cuidado da saúde da pessoa idosa, que levam em consideração o envelhecimento e o processo saúde/doença, promovendo o cuidado, a corresponsabilidade e a coparticipação.

Com isso percebe-se a importância de entender a (bio)gerontotecnologia como um elemento constituinte do arcabouço teórico, pois, segundo Burlá e Azevedo (2006), o aumento da expectativa de vida é uma das maiores conquistas biotecnológicas na área da saúde, resultado da redução dos índices da mortalidade e de uma melhoria das condições de vida em geral. O viver mais é acompanhado do declínio fisiológico das funções orgânicas e, consequentemente, da maior probabilidade do surgimento de doenças crônicas, incapacitantes e não evolutivas.

Por sua vez, A5 salienta que novas gerontotecnologias devem ser construídas, com vistas a auxiliar os familiares/cuidadores de pessoas idosas no cotidiano de cuidados, em um cenário multiprofissional, no qual se construam diálogos de saberes entre a pessoa idosa, a família e os profissionais. Nesse sentido, o foco recai sobre a abordagem inter-transdisciplinar, isto é, sobre o diálogo entre os diferentes conhecimentos dos docentes e discentes dos cursos das áreas da saúde e dos familiares/cuidadores em busca de um objetivo comum: melhorar o cuidado e a saúde da pessoa idosa ou das famílias com doença de Alzheimer.

Ao se integrar as pesquisas de A4, e A5, aliadas aos estudos de A6, tem-se que a gerontotecnologia pode ser implementada tanto na promoção da saúde, quanto nas ações de prevenção e controle de doença em percurso, estreitando a interação do profissional com as pessoas idosas, sendo um campo de estudo interdisciplinar que compreende o estudo científico para o desenvolvimento de técnicas, produtos e serviços, baseados no conhecimento do processo de envelhecer.

Assim, gerontotecnologia educacional pode ser compreendida como um conjunto de conhecimentos, produtos, processos e estratégias que abre novas possibilidades no processo de ensino-aprendizagem, por meio da valorização das relações e interações entre o enfermeiro, o idoso e a família.

Segundo De Carvalho, De Oliveira e organizadores (2014), as tecnologias são processos concretizados a partir da experiência cotidiana do cuidar em saúde e algumas, derivadas de pesquisa para o desenvolvimento de um conjunto de atividades produzidas e controladas pelos seres humanos. Servem, portanto, para gerar e aplicar conhecimentos, dominar processos e produtos e transformar a utilização empírica, de modo a torná-la uma abordagem científica.

Nesse contexto, compreende-se que a Biotecnologia valoriza as atitudes e habilidades adquiridas ao longo da vida cotidiana da pessoa idosa sendo possível potencializar a produção ou a aquisição de dispositivos, produtos e instrumentos de cuidado.

Relaciona-se A7 à (bio)gerontotecnologia pois os autores consideram que, para construir diretrizes na provisão de cuidados paliativos, é necessário e essencial um programa tecnológico e 
geriátrico-gerontológico bem-sucedido integrado, uma vez que proporciona a melhoria da qualidade de vida da pessoa idosa e de sua família.

Cabe salientar que, segundo Almeida Queiroz et al. (2018), é fundamental preparar a equipe multiprofissional no tocante aos cuidados paliativos para além das competências humanas, incluindo também as tecnológicas. Sob essas considerações, Gulini et al. (2018) alegam que a avaliação e a abordagem paliativista deve ser humana e tecnológica desde o momento da admissão da pessoa idosa e de sua família nos serviços de saúde, a fim de que se possa estabelecer limites terapêuticos e evitar falsas esperanças aos familiares e à equipe que assiste esse paciente.

Logo, a (bio)gerontotecnologia contribui para a reflexão da equipe multidisciplinar acerca da necessidade de integrá-la na promoção de cuidados paliativos da pessoa idosa, aliando-a à família do idoso, uma vez que efetiva e valoriza o ser humano e a tecnologia.

\section{CONSIDERAÇÕES FINAIS}

Ao conhecer quais as produções científicas da literatura nacional e internacional existem acerca da gerontotecnologia na promoção dos cuidados paliativos da pessoa idosa, compreende-se que é preciso criar uma cultura de cuidados paliativos para melhorar a qualidade de vida, promovendo o suporte clínico, humano e emocional. Além disso, é preciso realizar o controle de sinais e sintomas, comunicação terapêutica, atuar em equipe multidisciplinar pautada em princípios da bioética, com respeito à autonomia da pessoa idosa de maneira humanizada.

Com isso a (bio)gerontotecnologia constitui-se de um conjunto de áreas do conhecimento, as quais se inserem na promoção dos cuidados paliativos da pessoa idosa, já que a tecnologia alinha-se à efetividade do cuidado humanizado em prol do ser humano. Dessa forma, o grande potencial para inovação da gerontotecnologia e a crescente difusão dos cuidados paliativos leva a crer que a (bio) gerontotecnologia é uma ferramenta importante para o cuidado paliativo, sendo possível potencializar a produção ou a aquisição de dispositivos, produtos e instrumentos de cuidados.

\section{REFERÊNCIAS}

ALMEIDA QUEIROZ, Terezinha et al. Cuidados paliativos ao idoso na terapia intensiva: olhar da equipe de enfermagem. Texto \& Contexto Enfermagem, v. 27, n. 1, 2018.

BARROS, Edaiane Joana Lima et al. Gerontotecnologia educativa voltada ao idoso estomizado à luz da complexidade. Revista Gaúcha de Enfermagem, v. 33, n. 2, p. 95-101, 2012.

BOUMA, Herman et al. Gerontechnology in perspective. Gerontechology, v. 6, n. 4, p. 190-216, 2007. 
BURLÁ, Claudia; AZEVEDO, Daniel Lima. In: FREITAS, E. V. et al. (Eds.). Tratado de geriatria e gerontologia. Rio de Janeiro: Guanabara-Koogan; 2006. p. 1079-89.

CARVALHO, Aline Tomaz de; DE OLIVEIRA, Mariana Gonçalves. Nietsche EA, Teixeira E, Medeiros HP, organizadores. Tecnologias cuidativo-educacionais: Uma possibilidade para o empoderamento do (a) enfermeiro (a)? Porto Alegre (RS): Moriá; 2014. Revista da Rede de Enfermagem do Nordeste, v. 15, n. 1, p. 185-186, 2014.

CIOSAK, Suely Itsuko et al. Senescência e senilidade: novo paradigma na atenção básica de saúde. Revista da Escola de Enfermagem da USP, v. 45, p. 1763-1768, 2011. Disponível em: https://bit.ly/ 2xhSUBp. Acesso em: 11 abr. 2019.

FLORES, Vanessa Boeira; BENVEGNÚ, Luís Antônio. Perfil de utilização de medicamentos em idosos da zona urbana de Santa Rosa, Rio Grande do Sul, Brasil. Cadernos de Saúde Pública, v. 24, p. 1439-1446, 2008.

FORBES, Sarah; BERN-KLUG, Mercedes; GESSERT, Charles. End-of-life decision making for nursing home residents with dementia. Journal of Nursing Scholarship, v. 32, n. 3, p. 251-258, 2000.

GULINI, Juliana El Hage Meyer de et al. Predictors of death in an Intensive Care Unit: contribution to the palliative approach. Revista da Escola de Enfermagem da USP, v. 52, 2018. DOI: https://bit.ly/ 2Tu5B4L

GUTIERREZ, Beatriz Aparecida Ozello; DE BARROS, Thabata Cruz. O despertar das competências profissionais de acompanhantes de idosos em cuidados paliativos. Revista Kairós: Gerontologia, v. 15, p. 239-258, 2012.

MELNYK, Bernadette Mazurek; FINEOUT-OVERHOLT, Ellen (Ed.). Evidence-based practice in nursing \& healthcare: A guide to best practice. Lippincott Williams \& Wilkins, 2005.

MINAYO, Maria Cecília de Souza. Análise qualitativa: teoria, passos e fidedignidade. Ciência \& saúde coletiva, v. 17, p. 621-626, 2012.

ROTHER, Edna Terezinha. Revisão sistemática X revisão narrativa. Acta paulista de enfermagem, v. 20, n. 2, p. v-vi, 2007. 
SANVEZZO, Vitória Marques de Sá; MONTANDON, Diego Santiago; ESTEVES, Larissa Sapucaia Ferreira. Instruments for the functional assessment of elderly persons in palliative care: an integrative review. Revista Brasileira de Geriatria e Gerontologia, v. 21, n. 5, p. 604-615, 2018.

SILVEIRA, Maria Helena; CIAMPONE, Maria Helena Trench; GUTIERREZ, Beatriz Aparecida Ozello. Percepção da equipe multiprofissional sobre cuidados paliativos. Revista Brasileira de Geriatria e Gerontologia, v. 17, n. 1, p. 7-16, 2014. 